\title{
Automatic Phase-Detection and Identification by Full Use of a Single Three-Component Broadband Seismogram
}

\author{
by Chao-ying Bai* and B. L. N. Kennett
}

\begin{abstract}
Automatic-time picking continues to be a significant issue in seismogram analysis. Any individual method is based on separation of signal and noise in a particular domain and cannot ensure consistent onset time picking under all source, receiver, path, and noise conditions. However, a combination of three methods based on differing aspects of seismograms can be used to provide automated and consistent phase detection and interpretation using a single three-component record. For arrival detection, a combination of energy analysis, differences in instantaneous frequency, and an auto-regressive representation of the seismic record are used to develop an algorithm for robust phase extraction, suitable for use in real time. The character of the phases is extracted using polarization analysis using complex traces to separate $S$ - and $P$-wave phases. The combination of four different methods offers a very effective technique for automatic arrival detection and association from a single three-component broadband seismogram and from thence an approximate event location procedure.
\end{abstract}

\section{Introduction}

All the current methods of onset-time picking are based on recognizing the different character of signal and noise in a variety of domains, for example, amplitude (energy content), frequency (frequency content), waveform (similarity of waveforms), angle (differences in wave vector), and dynamic features (e.g., polarization, rectilinearity, power, and frequency spectrum). However, there is not a single method that can ensure consistent onset-time picking for every arrival. Any particular method will fail when the difference between a certain part of the noise and the signal is small, particularly when the signal-to-noise ratio $(\mathrm{S} / \mathrm{N})$ is low. For example, the energy approach, which is commonly the most useful, cannot be used when the noise level is high and later phases are buried in the coda of an earlier event. Bandpass filtering will not work when the noise and the signal have almost the same frequency content. In order to overcome the disadvantage of a single method, we seek to combine several methods so as to ensure consistent time picks.

Previous studies of arrival detection and recognition have generally been directed to a single phase and deal only with short-period seismograms. The principal aim has commonly been to pick a second arrival automatically following a first arrival with a clear time pick. With the advent of digital broadband seismology, the need to make full use and to provide onset-time estimates for several phases in the same record has become both urgent and necessary in the

*On leave from Xinjiang Seismological Bureau, Urumqi, China. context of the efficient exploitation of sparse networks such as the International Monitoring System of the Comprehensive Test Ban Treaty.

The methods commonly used for onset-time picks include energy analysis, polarization analysis, and auto-regressive techniques combined with prefiltering of records or transformation of data, for example, by removal of free surface effects. Earle and Shearer (1994) have used an energy method to deal with the records from the Global Digital Seismic Network and compared the automated onset-time picking with analyst time picks previously reported to NEIC. Tong and Kennett (1996) also used the energy technique to identify and pick the later phases of broadband records. Vidale (1986) has used polarization analysis applied to complex traces to recognize Rayleigh and Love waves, whereas Aster et al. (1990) used a polarization filter to highlight the direction of linear ground motion so as to produce a linearity-enhanced seismogram. Wagner and Owens (1996) have proposed the use of the principal eigenvalue in the frequency domain as a detector. Takanami and Kitagawa $(1988,1993)$ have used an autoregressive technique to pick secondary arrivals in a regional seismic network in Japan, and Leonard and Kennett (1999) have developed autoregressive methods for three-component analysis of broadband seismograms. Withers $e t$ al. (1998) have systematically compared several trigger algorithms currently used for onset-time picking and gave details of comparisons in the time and frequency domains and also for particle motion and adaptive processing. 
Such comparisons are useful for improving both the algorithms and the particular methods.

For a sparse network, we need to be able to exploit the full information content of single station data so that we can automatically pick several arrivals and provide a likely phase association. With a number of identified phases we can then attempt a single station location.

In this article, we attempt to follow the precept "the less filtering, the better the algorithm" (Douglas, 1997), and so we combine a variety of techniques that can be applied to unfiltered records without rotation. For arrival recognition and detection, we combine three different methods so as to ensure consistent onset-time picks. Even though a certain method fails because the noise characteristics are unfavorable, another may well be unaffected and the combination can achieve a satisfactory pick.

For a location estimate with a single station, we must first be able to pick at least four phases from the same record (we would prefer two $P$ and two $S$ arrivals), and second be able to distinguish $S$-wave phases from $P$-wave phases among the detected arrivals. With an $S$-wave detection and an azimuth measurement, we would have sufficient information to make an epicentral estimate using travel-time information.

\section{Brief Description of Methodology}

\section{Phase Detection}

We select three methods for estimating the onset time of arrivals that have different dependence on noise and signal content, and then combine the result to derive an onsettime pick. The methods we use are energy analysis, instantaneous frequency, and an autoregressive representation of the trace (cf. Bai and Kennett, 1999).

Energy Analysis. Phase detection using energy analysis is based on the ratio of a short-term estimate of the energy content to a long-term-average energy level (STA/LTA). Several different algorithms have been employed for this purpose. The differences among them have been choice of the energy ratio between different components and the use of different coordinate systems, and also the style of time window exploited when calculating the energy ratio. We choose the following combinations of energy

$$
\begin{array}{ll}
E_{3} & =Z^{2}+N^{2}+E^{2} \\
\text { the total energy } & Z_{\mathrm{e}}=Z^{2} \\
\text { the vertical energy } & \\
\text { the energy in the horizontal plane } H_{\mathrm{e}} & =N^{2}+E^{2}
\end{array}
$$

where $Z, N$, and $E$ are the amplitude of the three unrotated components. Note that these three energy measures are invariant under rotation. Following Tong and Kennett (1996), we use a sequential update procedure for the energy measures rather than a sliding window because this provides a very effective detection procedure and is readily adapted to real-time applications.

Auto-Regressive Representation. Broadband seismograms contain both the desired seismic signals and other unwanted contributions that we refer to as noise. For the seismic signal, we assume that each phase has its own characteristics (energy, frequency, waveform, and angle of vector) and will last at least a few seconds; thus in the presence of a seismic phase we expect that the present value of ground motion will be related to the previous value.

Leonard and Kennett (1999) have shown how the differences in the nature of the autoregressive representation of signal and noise can be used to make a separation of phase contributions from the background and thence make onsettime detections. For truly random white noise, the current value of ground motion is unrelated to the previous value, whereas microseismic noise such as the noise from distant storms or the unrecognized coda of an earlier event will have a weak association of present value with the previous value (which will be expressed by low-order correlation).

There are two ways to use an AR Model for phase recognition and onset-time picks. One approach is to use the Akaike Information Criterion (AIC) (Akaike, 1973) to determine the order of an AR filter and apply the AR filter to seismograms, and then recognize onset-time picks as the point where the style of AR representation changes (Takanami and Kitagawa, 1988; Leonard and Kennett, 1999); the other approach is to use a predictive AR model to separate the seismic signal from background noise (Takanami and Kitagawa, 1993). For recognition and combination, we use a simple but useful approach and construct just the AR tensor coefficients from three-component records and assume that the values of the AR coefficients will be augmented when a seismic phase arrives.

The tensor AR coefficients have been estimated using a 9th order AR representation constructed from a long retrospective window which is successively advanced by $2 \mathrm{sec}$ intervals. In order to generate a set of scalar measures from the tensor AR coefficients, we have summed the absolute values of the the auto- and cross-correlation coefficients for each order from 1 to 9 . Then to provide an indication of the change in character of the AR coefficient patterns, we have summed over three orders at a time to produce a set of summary attribute estimates. We construct a low order attribute (AR1) by combining the coefficients from order 1 to 3 , a medium order attribute (AR2) from orders 4 to 6 , and the high-order correlation attribute (AR3) from orders 7 to 9 . The combination of the different tensor coefficients into summary measures minimizes the dependency of the results on the orientation of the horizontal components, and we have found little difference between rotated and unrotated records.

We anticipate that the different styles of signal and noise will be reflected in the time variation of these summary mea- 
sures with short-period signal most noticeable in the highorder coefficients. The AR measures are generated continuously along the trace, and phase recognition or detection scheme is based on the use of a STA/LTA ratio technique. We declare a detection trigger for a measure such as AR3 when the ratio of the STA to LTA values for AR3 surpasses a predetermined value.

Instantaneous Frequency Technique. We anticipate that there will be a difference in both phase angle and frequency between signal and noise. This difference is exploited by introducing instantaneous frequency analysis using complex traces. The three components of a seismic trace can be converted to an analytic signal via:

$$
\vec{u}_{j}(t)=u_{j}(t)+i \mathcal{H} u_{j}(t), \quad j=1,2,3
$$

where $u_{j}(t)$ is the unrotated real seismic record, $\mathcal{H} u_{j}(t)$ is its Hilbert transform, and $\vec{u}_{j}(t)$ is the associated complex trace. A number of different formulations of instantaneous phase angles can be made depending on whether we work with one, two, or three-components (Bai and Kennett, 1999). We select a number of different definitions for instantaneous phase angles:

a. Instantaneous phase in two dimensions:

$$
\alpha_{i j}=\tan ^{-1}\left(\frac{\sqrt{\left(\mathcal{H} u_{i}\right)^{2}+\left(\mathcal{H} u_{j}\right)^{2}}}{\sqrt{u_{i}^{2}+u_{j}^{2}}}\right)
$$

where $i, j=1,2,3$ corresponding to $Z, N$, and $E$ components, but $i \neq j$.

b. Instantaneous phase in three dimensions:

$$
\alpha=\tan ^{-1}\left(\frac{\sqrt{\left(\mathcal{H} u_{1}\right)^{2}+\left(\mathcal{H} u_{2}\right)^{2}+\left(\mathcal{H} u_{3}\right)^{2}}}{\sqrt{u_{1}^{2}+u_{2}^{2}+u_{3}^{2}}}\right)
$$

c. Instantaneous phase angle between a real two-dimensional vector and an imaginary three-dimensional vector:

$$
\alpha_{i j}(I)=\tan ^{-1}\left(\frac{\vec{u}_{i j}}{\mathcal{H} \vec{u}}\right)
$$

d. Instantaneous phase angle between an imaginary twodimensional vector and a real three-dimensional vector:

$$
\alpha_{i j}(R)=\tan ^{-1}\left(\frac{\mathcal{H} \vec{u}_{i j}}{\vec{u}}\right)
$$

With these definitions of the instantaneous phase angles we can further define an instantaneous frequency through

$$
f(t)=\frac{\mathrm{d}}{\mathrm{d} t}(\alpha(t))
$$

where $\alpha(t)$ is one of the instantaneous phase angles previously defined. When we take account of the different combinations of the ground-motion components in the threecomponent records we produce a total of 10 different estimates of instantaneous frequency.

We also construct three different summary sets of frequency information designated as $F Q 1, F Q 2$ and $F Q 3$. $F Q 1$ is the sum of the instantaneous frequency associated with the phase angles (a) and (b) $(2.3,2.4) ; F Q 2$ is the sum of the frequencies associated with the phase angles of the form (c) (2.5), and FQ3 is the sum of frequencies related to the phase angles of the form (d) (2.6). The use of averaged frequency measures rather than a single frequency has distinct advantage of stability. We again use a similar recognition and declaration scheme for a phase onset.

The individual estimates of instantaneous frequency depend on the orientation of the components, but such influences are minimized when we combine the many different styles of frequency measures in the summary attributes. In continuous monitoring, the construction of the complex traces is probably simplest to accomplish with a overlapped set of fixed length windows and a Fast Fourier transform that will introduce a slight delay in processing.

\section{The Technique of Combination Analysis}

We have briefly summarized three different methods for extracting seismic phases based on recognizing the differing content of noise and signal in different domains, and we have further extracted three different measures from each method. For energy analysis, the three measures are the total energy $\left(E_{3}\right)$, the vertical energy $\left(Z_{e}\right)$, and the energy in the horizontal plane $\left(H_{e}\right)$; for instantaneous frequency analysis, the three measures are $F Q 1, F Q 2$, and $F Q 3$; for autoregressive coefficient analysis, the three estimates are the low-order correlation (AR1), the medium-order correlation (AR2), and the high-order correlation (AR3).

Using these nine attribute estimates from the three different methods, we construct an algorithm for phase recognition and onset-time picks. Firstly, for each of the attribute estimates from the different methods, we set a token equal to unity when the STA/LTA ratio surpasses a preset value, otherwise, the token is set equal to zero. Secondly, we declare a recognition or detection trigger when a combination of the nine attribute tokens within a $2 \mathrm{sec}$ window exceeds a prespecified value.

When we combine the different measures, we use two different procedures so that we can follow fine changes in the content of noise and signal. In the first approach (equal weighting) we place equal weight on each attribute and we are only concerned with the sum of the tokens rather than the contributions of different methods. In the second (equal content), we attempt to equalize the contribution from the 
different methods of phase assessment. Whichever process is used, we use a ternary representation for a phase detection and specify two different preset thresholds for the combination of the attribute tokens. The combination measure remains in state 0 until the lower threshold is exceeded when we represent the detection by a "small flag" (state 1) and when the higher threshold is also reached we set a "big flag" (state 2).

For the equal weighting procedure, we set the threshold for the "small flag" at 3 and the "big flag" at 6 . For the equal content combination, we set a "big flag" when there is some contribution from each of the three different methods and a "small flag" when any two different methods contribute. Finally, we declare a detection of a phase arrival when both of the schemes for combining the phase information tokens have a flag raised.

\section{Phase Characterization}

When we try to locate an event from a single threecomponent record, we need to be able to ensure consistent arrival picks and guarantee to pick no less than 4 arrivals, we will demonstrate that the combination of detection methods works well in this regard. A second problem is to be able to distinguish $S$-wave phases from $P$-wave phases, which can be achieved using polarization analysis.

From polarization analysis of complex traces, we extract at least seven parameters such as the elliptical component of polarization $\left(P_{\mathrm{E}}\right)$, the linear polarization $\left(P_{\mathrm{S}}\right)$, the strike and dip of the direction of maximum polarization $(A Z I)$ and $(D I P)$, and the real part of the eigenvector $\left(P_{1}, P_{2}, P_{3}\right)$ associated with the largest eigenvalue that points in the direction of the largest amount of polarization. Detailed descriptions of such polarization analysis for complex traces are discussed by Vidale (1986) with further applications in Bai and Kennett (1999).

Separating Body Waves from Background Noise. Body waves have linear polarization, and if this property can be recognized, we can use polarization results to separate body waves from background noise. Theoretically, we can anticipate a relatively stable strike and dip of the direction of maximum polarization when a seismic phase arrives. Thus, we can separate the seismic-wave segment from background noise by looking for a low gradient of the strike

$$
\frac{\mathrm{d}}{\mathrm{d} t} A Z I(t)=\left|A Z I\left(t_{1}\right)-A Z I(t)\right| \leq C_{0}
$$

where $A Z I(t), A Z I\left(t_{1}\right)$ are the estimates of the strike at successive time samples, and $C_{0}$ is a preset threshold.

Further, from the definitions of angular and linear polarization, the strength of linear polarization will be strong when the angular polarization is small and linear polarization is large. Thus, we can construct a measure of enhanced linear polarization when

$$
B W P=\Delta P=\left|P_{\mathrm{S}}(t)-P_{\mathrm{E}}(t)\right| \geq D_{0}
$$

where $P_{\mathrm{E}}(t)$ and $P_{\mathrm{S}}(t)$ are the elliptical and linear polarization at time $t$, and $D_{0}$ is a preset value. It is possible to use $P_{\mathrm{S}} /$ $P_{\mathrm{E}}$ as the measurement of enhanced linear polarization, but we use $B W P$ because it is still effective when both $P_{\mathrm{E}}$ and $P_{\mathrm{S}}$ have a large value.

We use a combination of these two linear polarization measures to determine the likely presence of a body wave.

Distinguishing $S$ Waves from $P$ Waves. When an $S$ wave arrives, we expect that $D I P$ will be small and stable, and for a $P$ wave that the $P_{1}$ component of the real-part of the eigenvector will be the most important.

Based on these observations, we can define two nonequivalent formula for each of $P$ - and $S$-wave recognition from the elements of the polarization eigenvector $\left(P_{1}, P_{2}\right.$, $\left.P_{3}\right)$

$P$ waves

$$
\begin{gathered}
P W D 1=\frac{P_{1}^{2}}{P_{1}^{2}+P_{2}^{2}+P_{3}^{2}} \geq C_{1} \\
P W D 2=\cos ^{-1}\left(\frac{P_{1}}{\sqrt{P_{1}^{2}+P_{2}^{2}+P_{3}^{2}}}\right) \geq C_{2}
\end{gathered}
$$

$S$ waves

$$
\begin{gathered}
S W D 1=D I P \leq D_{1} \\
S W D 2=\frac{P_{2}^{2}+P_{3}^{2}}{P_{1}^{2}+P_{2}^{2}+P_{3}^{2}} \geq D_{2}
\end{gathered}
$$

where $C_{1}, C_{2}, D_{1}, D_{2}$ are the preset thresholds related to each of the detection techniques.

The use of the analytic trace, rather than just the real part of the signal, has two main advantages: firstly, that the polarization can be measured from the covariance at any point in the seismogram, and secondly, the complex eigenvector is rotated to the direction of the largest amount of polarization, which is better than the use of real analysis for the process of separating $P$-wave energy, which is mainly on vertical component, from the $S$ energy, which lies chiefly in the horizontal plane.

Event Location using a Single Three-Component Record. If we are able to recognize at least four phases and to provide a clear wavetype association, we are in a position to estimate the location of the source. The simplest approach is to assume a shallow source and use the differential time between the different phases to estimate the epicentral distance and then find the location by adding in an azimuth estimate. The depth can be refined to some extent by using the differential time between $P$ and $S$ (Tong and Kennett 1996). An ap- 
proximate epicentral distance will help with phase identification and may also allow the recognition of depth phases.

In the presence of significant noise, we are likely to produce a number of false phase detections and will then need to use all the available information on the likely character of a phase to sift out the unlikely phase combinations. This will still lead to a number of different epicentral estimates (especially since the azimuth is also likely to be contaminated). In this case, the choice between different locations will only be feasible if additional information can be included, for example, location estimates from a second station.

\section{Trace Analysis and Discussion}

We present a comparison of the performance of the different styles of trace analysis on six examples taken from four events in different locations, which have been recorded on a portable broadband station in different parts of Australia. These sets of broadband records show a variety of noise conditions, S/N, and different paths and locations, and enable us to examine the value of the processing techniques in a range of environments from far regional to teleseismic distances with different event orientations. The use of multiple records from the same events as well as records from different events allow us to look at both the similarities and differences in the performance of the algorithms at different stations.

The four events (Table 1) cover the depth range from shallow to intermediate depth in a variety of locations in the earthquake belts to the north and east of Australia. The 6 records we display cover an epicenter range from $17^{\circ}$ to $42^{\circ}$, with azimuths from $35^{\circ}$ to $350^{\circ}$. To compare the performance of the algorithm, we have selected four sets of unrotated records and two records that have been rotated to the great-circle $(Z, R, T)$ for the particular paths. The locations of the events and stations and the great circle paths are displayed in Figure 1.

\section{Analysis of Results}

In order to ensure consistent onset-time picks, we try to maintain a constant set of prespecified thresholds. For energy analysis, the thresholds is set at 2.2; for instantaneous frequency and autoregressive coefficient analysis, we nor- mally use a threshold of 1.25 . In order to separate the successive arrivals, we take a short-term average over a window $2 \mathrm{sec}$ long, the long term window is usually set at $62 \mathrm{sec}$ for energy analysis and $31 \mathrm{sec}$ for both frequency and AR coefficient measures. We set the token for a particular measure equal to unity when the STA/LTA ratio exceeds the appropriate threshold, otherwise the token is set equal to zero. The combination of the tokens for the different techniques are then used as discussed in section 2.2, with a declaration of a detection of a phase arrival when both of the schemes for combining tokens have a flag raised.

For the process of distinguishing different types of waves, we declare a separation of a body wave from background noise when both (2.8) and (2.9) are satisfied, and declare a recognition of $P$ or $S$ wave among the detected arrivals when one of the recognition schemes for $P$ or $S$ waves $(2.10-2.13)$ is satisfied. The polarization measures have been extracted with a constant window length of $2 \mathrm{sec}$ for all the examples.

The process for automatic-onset detection and identification of wave type can be summarized as

$$
\begin{gathered}
\text { arrival detection } \Rightarrow \text { body-wave identification } \Rightarrow \\
\text { wave-type recognition. }
\end{gathered}
$$

A summary of the attribute measures displayed in the figures is provided in Table 2.

In the following examples, we make a distinction between the cases where the combination algorithm indicates the presence of phases which can be identified using the iasp 91 travel times and the parameters for the event. These are designated as phases $1,2, \ldots n$ and marked in the figures by a long black vertical bar. Where the combination algorithm produces a detection without a clear phase association we denote the detections as arrivals $1,2, \ldots n$; these are marked with a shorter vertical bar in grey.

Event 1. We present the analysis of two records at stations SA06 and SA08 in eastern Australia in Figures 2 and 3. These records lie in the far-regional range and have a smaller epicentral distance than the other examples. We display the results from the nine individual measures together with the different styles of combination measures COM (equal weighting) and CM (equal content). The big flag detections are indicated by a taller bar than the small flag detections.

Table 1

The Parameters of the Records for the Four Events

\begin{tabular}{cccccccccc}
\hline Event No. & Time & Code & Lat. & Long. & Depth & Mag. & Az. & Del. & Data \\
\hline 1 & 93.134 .21 & SA06 & -10.82 & 161.36 & 25.0 & 5.3 & 48.22 & 17.31 & UR \\
1 & 93.134 .21 & SA08 & -10.82 & 161.36 & 25.0 & 5.3 & 35.69 & 17.82 & UR \\
2 & 94.003 .13 & SB02 & -49.27 & 164.22 & 16.0 & 0.0 & 149.61 & 28.11 & R \\
3 & 94.019 .01 & SB03 & -3.24 & 136.00 & 29.2 & 0.0 & 350.11 & 25.55 & R \\
4 & 95.084 .22 & SD09 & -11.00 & 166.12 & 79.0 & 5.9 & 57.75 & 31.44 & UR \\
4 & 95.084 .22 & SD03 & -11.00 & 166.12 & 79.0 & 5.9 & 75.44 & 41.64 & UR \\
\hline
\end{tabular}




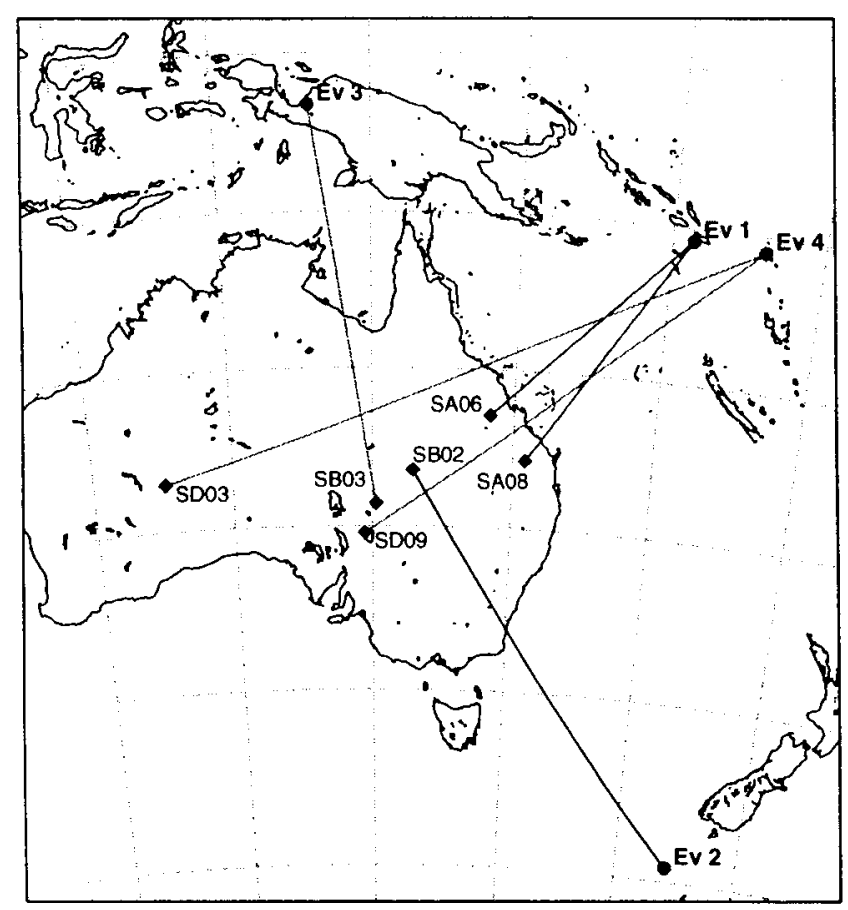

Figure 1. Events, stations and great circle paths used in the examples.

Table 2

The Attributes Used for Phase Detection

\begin{tabular}{cclc}
\hline Method & Attribute & \multicolumn{1}{c}{ Character } & Equation \\
\hline Energy & $E_{3}$ & Total Energy & $(2.1)$ \\
Analysis & $Z_{e}$ & Vertical Energy & $(2.1)$ \\
& $H_{e}$ & Energy in the horizontal plane & $(2.1)$ \\
Instantaneous & $F Q 1$ & Sum of 2-D and 3-D & $(2.3),(2.4)$, \\
Frequency & & measures & $(2.7)$ \\
& $F Q 2$ & Sum of frequency measures & $(2.5),(2.7)$ \\
& $F Q 3$ & Sum of frequency measures & $(2.6),(2.7)$ \\
Auto- & $A R 1$ & Sum over AR orders 1-3 & \\
Regressive & $A R 2$ & Sum over AR orders 4-6 & \\
Analysis & $A R 3$ & Sum over AR orders 7-9 & \\
Composite & $C O M$ & Equal weighting & \\
Measures & $C M$ & Equal content & \\
Polarization & $A Z I$ & Stability of strike of eigenvector & $(2.8)$ \\
Measures & $B W P$ & Linearity of polarisation & $(2.9)$ \\
& $P W D 1$ & P detector-eigenvector properties & $(2.10)$ \\
& $P W D 2$ & P detector-eigenvector properties & $(2.11)$ \\
& $S W D 1$ & S detector-dip of eigenvector & $(2.11)$ \\
& $S W D 2$ & S detector-eigenvector properties & $(2.12)$ \\
\hline
\end{tabular}

From Figure 2, it is obvious that the performance of the combination algorithm is better than the performance of any single method. For example, the energy method can only pick the strong seismic phases $(P n, P g P g$, and $S n S n)$ by means of energy content if at least two attributes have a raised flag; the instantaneous frequency approach not only can pick strong phase ( $P n$ and $S n S n)$, but also can indicate the weak seismic phase $(P c P)$ not indicated by energy analysis, the use of the AR coefficients can pick nearly all the phases ( $P n, P n P n, P g P g, S n S n$, and $P c P$ ) which are indicated by using the iasp 91 travel-time tables (Kennett and Engdahl, 1991). The combination algorithm can also separate successive arrivals (such as $P n$ and $P n P n$ ).

For those detected arrivals that are indicated by traveltime tables, phases 1,2 , and 3 can be distinguished as $P$ wave arrivals with the linear polarization property of body wave; phase 4 can not be definitely identified as $S$-wave phase due to uncertainty in the polarization features of the body wave. Phase 5 has also uncertain style of $P$ or $S$ wave, because it is immersed in the $S$-wave segment. However the $P$ and $S$-wave segments of the record can be clearly separated from the behavior of the measures PWD1, PWD2, and SWD1, SWD2. Besides these five main phases, there are four other detections that do not correspond to phases expected from the iasp 91 travel-time tables using the event parameters.

Figure 3 shows another record for event 1 . In this case, the performance of the combination algorithm is striking and is better than the performance of any single method. Once again phases 1 and 2 can be recognized as $P$-wave arrivals, phases 3 and 5 as $S$ waves, but surprisingly we correctly recognize arrival 4 as a $P$-wave phase even though it lies within the $S$-wave train. There are also two other detected phases that were not indicated by iasp 91 travel-time table for the event parameters. We note that the arrivals suggested from the combination procedure even though they do not correspond to recognized phases occur at about the same time intervals after the first arrivals $(40,155,350 \mathrm{sec})$ and could well be associated with another event.

Event 2. We now consider an event in the near teleseismic distance range with a relatively high noise level associated with a very intense summer storm off the costs of Southeast Australia with complex microseisms. In this case we consider the use of the combination algorithm for phase detection with records rotated to the great-circle using the information for the event. The $35^{\circ}$ rotation has little effect on the nature of the summary measures.

At first glance, we cannot definitely separate the $S$-wave segment from $P$ waves, and indeed it is quite hard to distinguish the first arrival of the $P$ and $S$ waves. We therefore have a strong test of the use of the combination technique. From Figure 4 we see that the energy analysis can only pick the large amplitude $P$ and $P g S(S P g)$ phases and fails to pick the other arrivals even though the data is rotated to the $Z, R$, $T$ coordinate system. In contrast the combination of the different methods can pick out five arrivals $[P, P c P, S, P g S$ $(S P g)$ and $S n S n]$, but there are also a number of other detections which do not coincide with expected arrivals. The first arrival of the $S$ wave is picked up by both the change in instantaneous frequency and the AR coefficients. The phases 1 and 2 can be distinguished as $P$ waves, and phases 3,4 , and 5 as $S$-wave phases. There are 7 other arrivals 


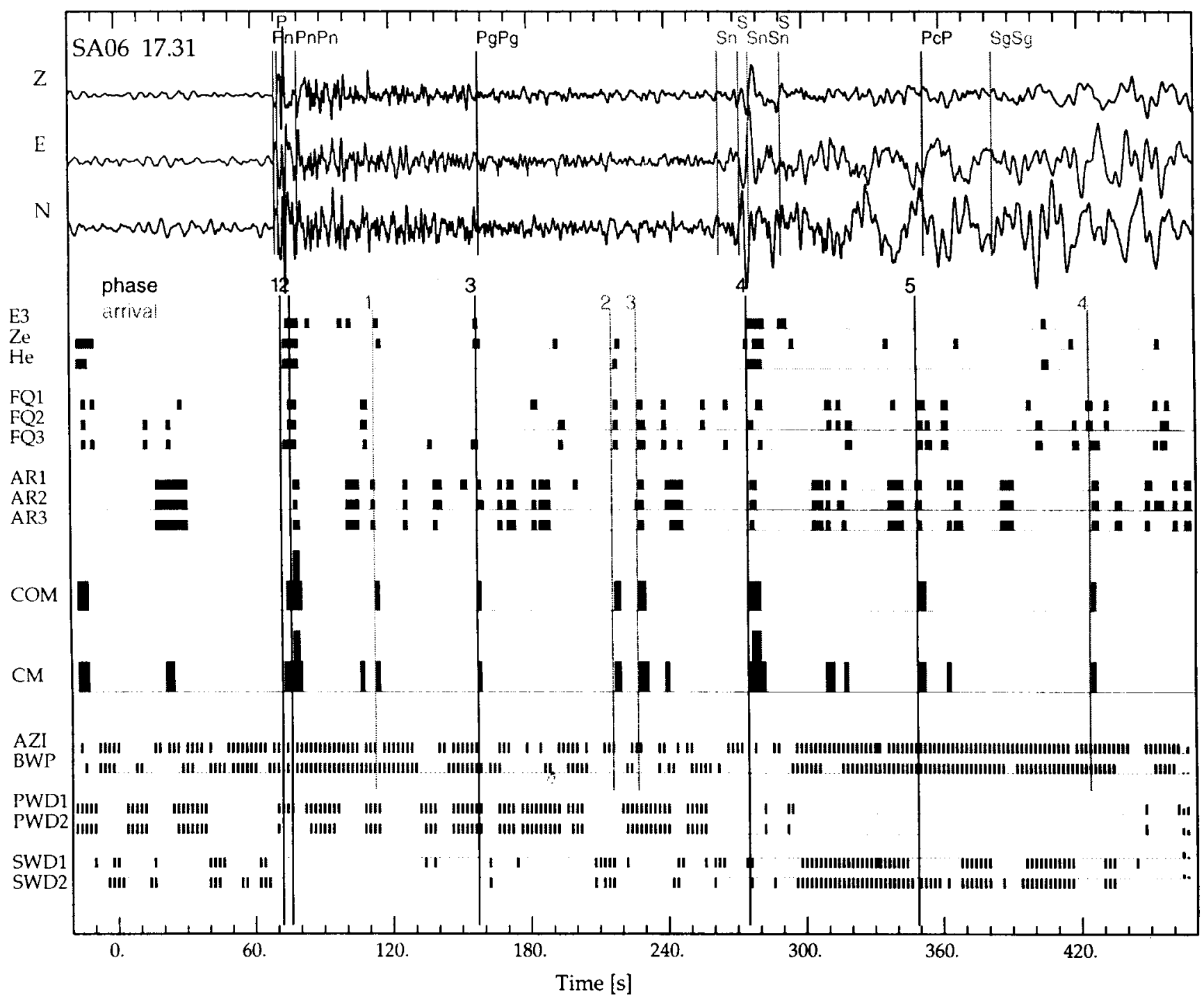

Figure 2. An example of the application of the combination algorithms to event 1 recorded at station SA06 at an epicentral distance of $17.31^{\circ}$. The short-term window for three different methods is $2 \mathrm{sec}$; the long-term window for energy analysis is $62 \mathrm{sec}$, and for frequency and coefficient analysis is $31 \mathrm{sec}$. The upper three traces represent the three-component seismic records without rotations, with the superimposition of the predicted times of arrivals using iasp 91 travel-time tables for the event parameters. $E_{3}, Z_{\mathrm{e}}, H_{\mathrm{e}}$ are the total, vertical, and horizontal energy ratio of current value to long-term trend. FQ1, FQ2, and FQ3 represent frequency ratios of the local value to the long-term trend, and AR1, AR2, and AR3 are the low-order, medium-order, and high-order correlation coefficient ratio of temporal behavior to long-term trend. COM represents the use of an equal content combination and $\mathrm{CM}$ the equal weighting procedure. $A Z I$ and $B W P$ combined forms a separation of body wave from background noise; $P W D 1$ and $P W D 2$ are detectors for $P$ phases, and $S W D 1$ and $S W D 2$ are the detectors for $S$ phases. The long black vertical bar reaching to the bottom of the frame represents the case where both the iasp 91 travel times and the combination algorithm both indicate a phase, denoted as phases $1,2, \ldots n$. A shorter vertical bar in grey indicates where only the combination algorithm indicates a detection, denoted as arrivals $1,2, \ldots n$.

arising from the contamination of the record by the coda of an earlier event.

In such a case the results from the combination analysis could help to guide an analyst in the recognition of the significant phases.
Event 3. Event 3 is slightly deeper than the previous events, and we consider the application of the combination algorithm to the record at station SB03 at an epicentral distance of $25.5^{\circ}$ (Figure 5). In this case we have used rotated seismograms, as the path is close to north-south, and we can 


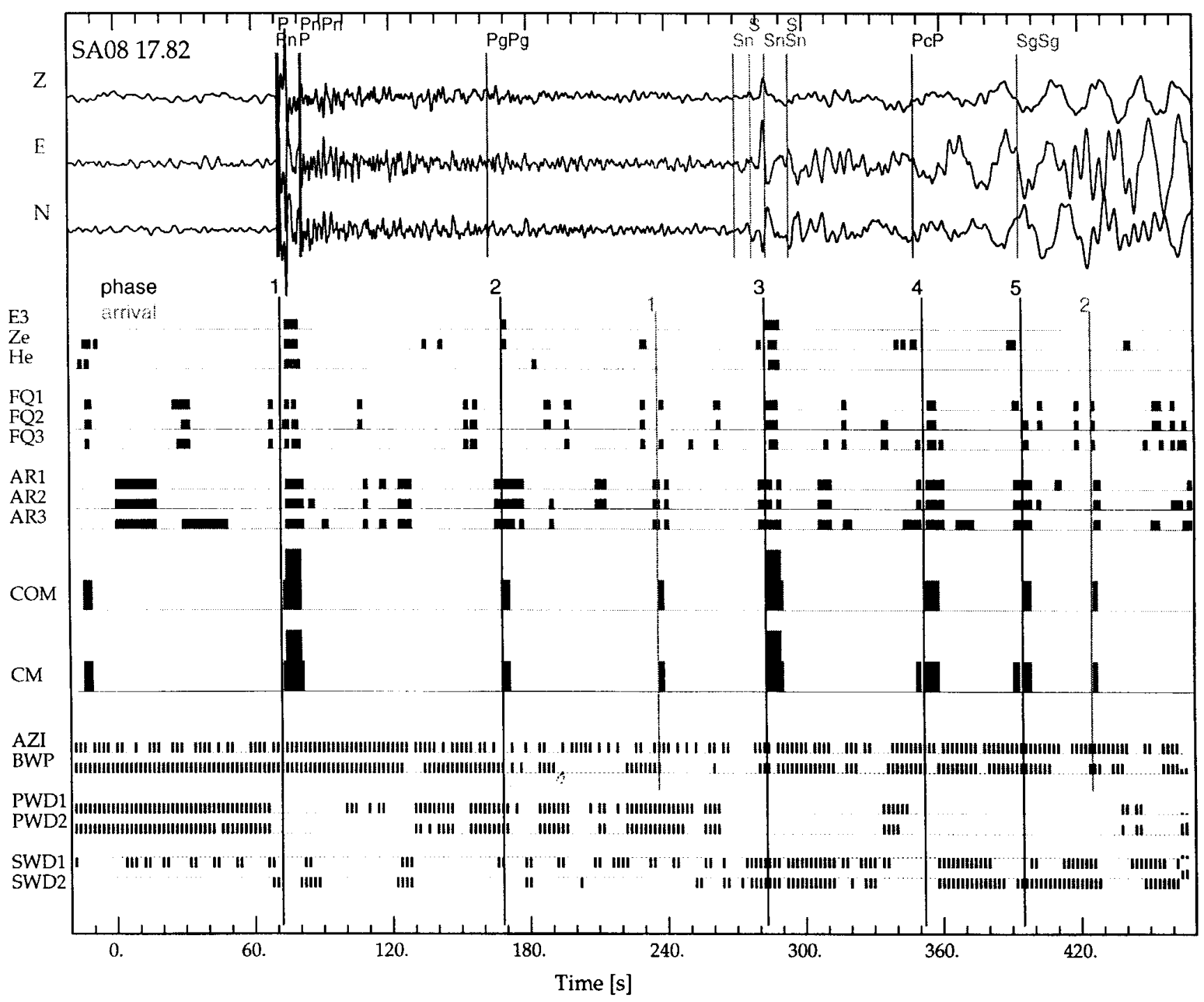

Figure 3. A further example of the application of the combination algorithms to event 1 for station SA08 at an epicentral distance of $17.82^{\circ}$. The traces follow Figure 1, with indications of clear phase detections (phases) and unassociated detections (arrivals).

see that the performance of combination algorithm is better than that of any single method. We would miss the first arrival of the $P$ wave and the depth phase $s S$ if we only used energy analysis.

The combination algorithm is able to pick up the groups of arrivals associated with the $P$ - and $S$-wave onsets and also to provide clear wave type associations for these phases. In this case we also note organized arrivals preceding the expected $P$ arrival and three other apparent phases that do not have the expected character of a body wave arrival or coincide with a recognizable arrival.

Event 4. As a further illustration we consider an intermediate depth event recorded in the near teleseismic range at stations in southern Australia with moderate noise level. Figure 6 shows the analysis for station SD09 at $31.44^{\circ}$ epicentral distance and Figure 7 shows the analysis for station SD03 at the greater distance of $41.64^{\circ}$.

At station SD09 (Fig. 6), all the arrivals that are expected from the iasp 91 travel-time tables can be detected by the combination algorithm. Phases 1, 2, 3, and 5 can be distinguished as $P$ phases, and phases 6,7 , and 9 as $S$ waves. This is a good example of the way in which the individual methods reinforce each other when used in combination. Energy analysis picks only $P, s P$, and $S$ phases; frequency analysis and AR coefficient analysis pick $P, p P, s P, P n P n, S, s S$ (or $S P g$ ), $S c P$, and $P c S$, but the significance of the picks are reinforced by the coincidence of the two methods.

At station SD03 (Fig. 7), the combination technique provides effective picks for all the main arrivals except for depth phase $s S$ and transform phase $S c P$, and also a clear wavetype attribution. The arrival 1 appears to be associated 


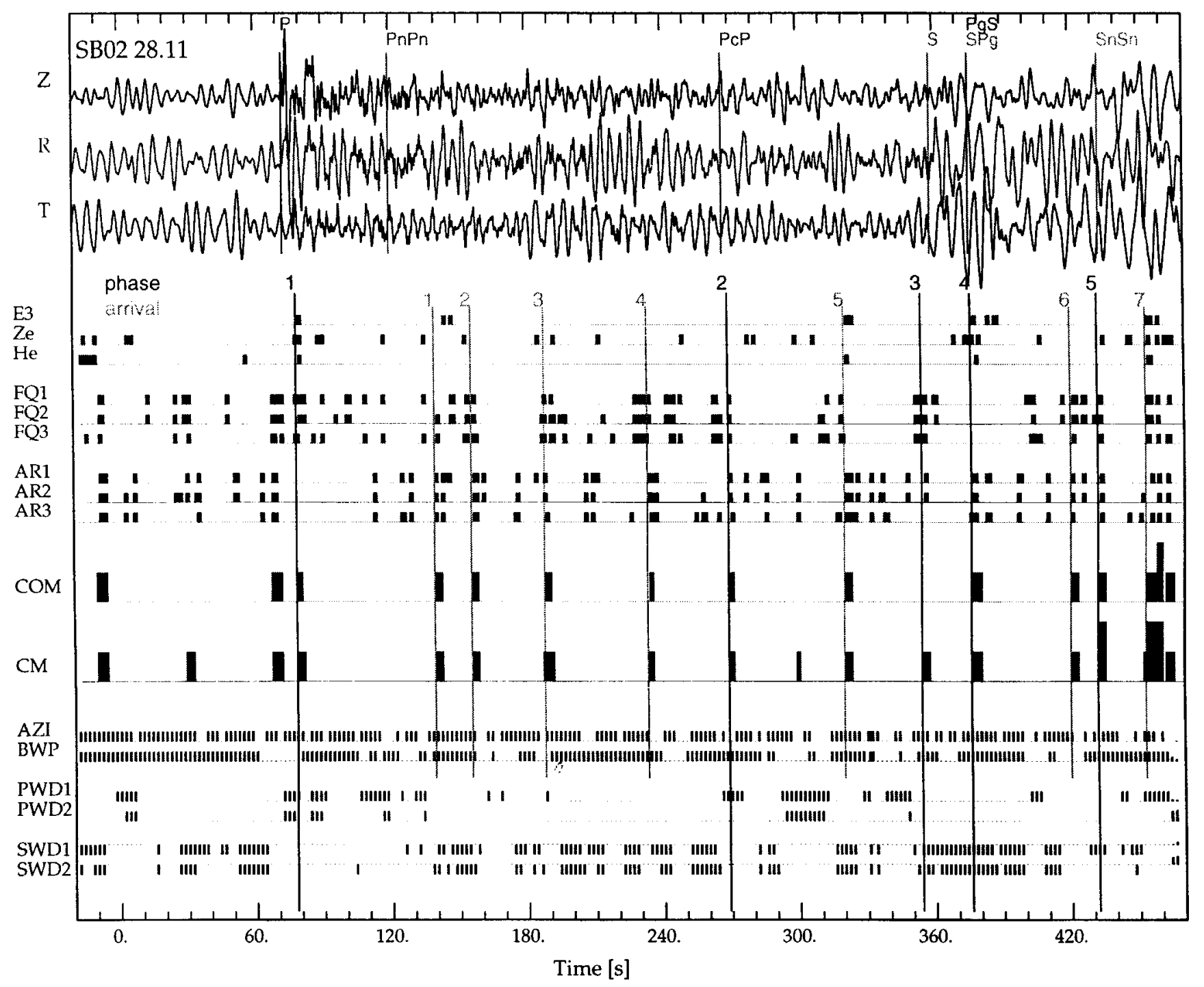

Figure 4. An example of the application of the combination algorithms to event 2 for station SB02 at an epicentral distance of $28.11^{\circ}$. The data is rotated to the Z,R,T data system. The upper three traces are vertical, radial, and tangential components with the indications of predicted times of arrivals using iasp91 travel-time tables. The other traces follow Figure 1, with indications of clear phase detections (phases) and unassociated detections (arrivals).

with the combination of an upgoing leg and multiple reflection branches through $p P P n, p P P$, which is enhanced because of a favorable position on the radiation pattern and the interaction of $P P$ triplications at $41^{\circ}$.

\section{Nature of Unassociated Detections}

In each of the examples above we have encountered a number of detections indicated as arrivals which do not have a clear connection to the phases predicted using the event parameters. Such additional detections tend to be more prevalent when the noise level is higher.

A few such detections are associated with less common seismic phases that are not included in the iasp 91 tabulations e.g. $P P P$ or the combination of a surface leg with multiple reflections, for example, $s P P$. A common cause is arrivals from overlapping events, for example, in the interval before a significant event we can find indications of smaller events which might not be expected to have been seen at the particular distance. Such detections are genuine phases and could be expected to be resolved by the different time relations and the various traces where recordings from a number of stations are available.

On occasion there is a clear change in the character of the record but no obvious phase present, such arrivals could be associated with scattering. 




Figure 5. An example of the application of the combination algorithms to event 3 for station SB03 at an epicentral distance of $25.55^{\circ}$. The data is rotated to the Z,R,T data system. The other traces follow Figure 1, with indications of clear phase detections (phases) and unassociated detections (arrivals).

\section{Conclusions}

Based on our knowledge that the character and attributes of signals and noise differ in energy content, frequency content, waveform correlation, and the polarization pattern of the wave vector, we have developed an algorithm for phase detection from a single three-component record that is suitable for real-time analysis. We declare a phase detection by the combination of results from multiple techniques with different sensitivity to noise. The method works successfully even when the $\mathrm{S} / \mathrm{N}$ is quite low and weak phases are buried in the coda of earlier events. The combination approach is built from a set of simple operations and a few threshold parameters that can be readily developed as data analysis proceeds. We have found that a standard combi- nation of thresholds and window parameters can be applied across a significant range of epicentral distances from farregional to teleseismic. The simplicity of the approach means that it can also be used in a multichannel situation such as the practical operation of a seismic network.

Polarization analysis has been quite successful in distinguishing body waves from background noise, and further, even in the presence of significant noise, it can provide a good indication of the general character of a portion of a record and so separate the $S$-wave train from $P$ waves.

The combination algorithm shows a good performance in picking up the first arrival associated with an event and also in resolving successive closely spaced arrivals. Consistent detection of the first arrival is important and necessary for both event location and later phase recognition. We have 


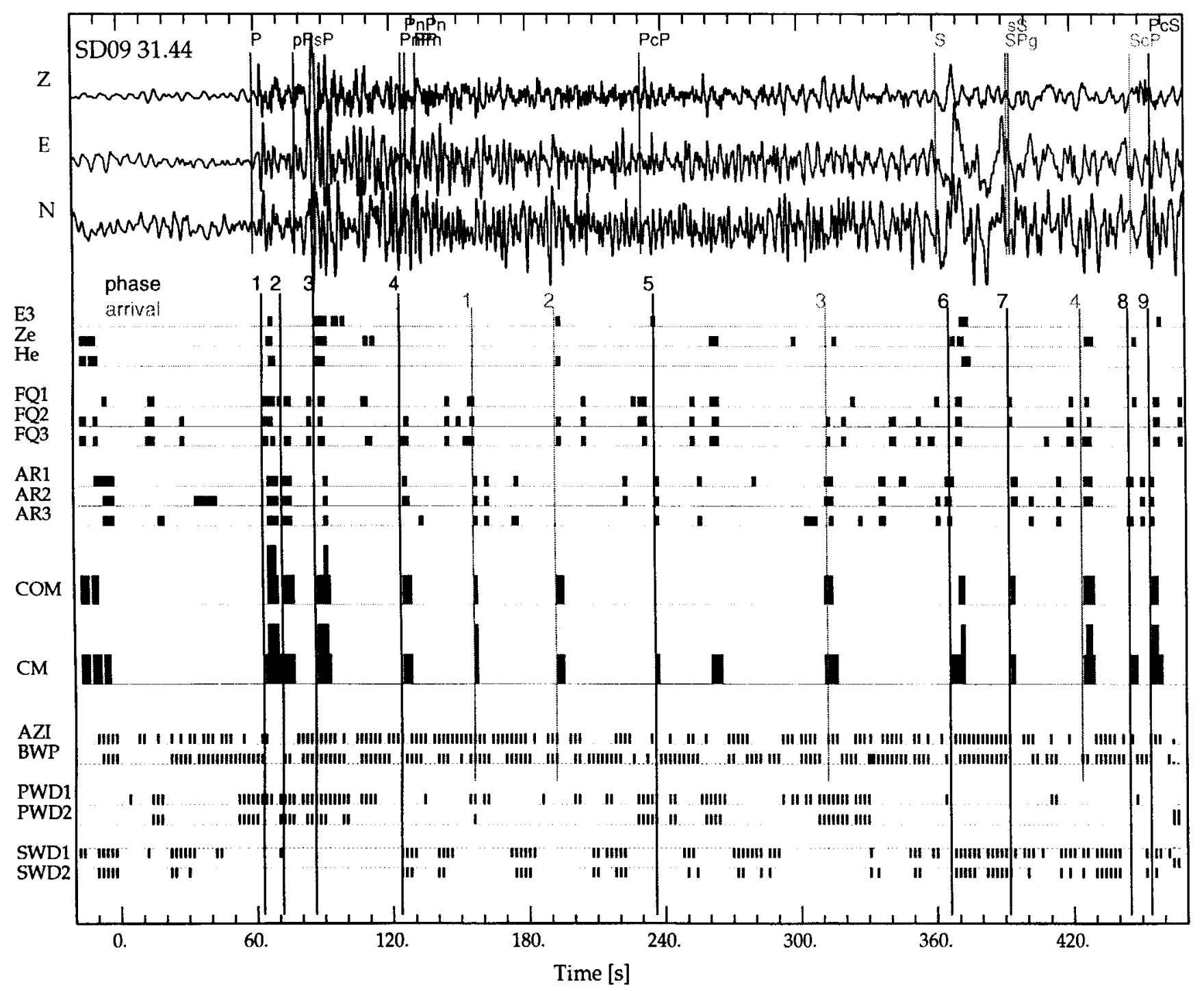

Figure 6. An example of the application of the combination algorithms to event 4 for station SD09 at an epicentral distance of $31.44^{\circ}$. The traces follow Figure 1, with indications of clear phase detections (phases) and unassociated detections (arrivals).

employed the procedure with equal weighting for the different approaches but it would be possible to apply different weightings to reflect the circumstances at particular stations or such effects as seasonal variations in the nature of the seismic noise.

The current set of windows and thresholds mean that the effective precision of the onset of a detection is about 1 sec and that differential times between $P$ and $S$ can generally be constrained to within $2 \mathrm{sec}$, which is equivalent to about $0.3^{\circ}$ in the distance range from $20-60^{\circ}$. This is a very useful result in the preliminary estimation of event location.

Once a phase detection has been made, it is possible to employ more sophisticated picking algorithms such as the tensor AR methods described by Leonard and Kennett (1999) . The merit of the present approach is that a variety of candidate phases are detected and assigned a character in a systematic procedure that still operates in high noise conditions on unfiltered records. Subsequent filtering can help to clarify the nature of phases.

\section{References}

Akaike, H. (1973). Information theory and extension of the maximum likelihood principle, in 2nd International Symposium on Information Theory, B. Petrov and F. Csaki (Editors), 267-281.

Aster, R., P. Shearer, and J. Berger (1990). Quantitative measurements of shear wave polarizations at the Anza seismic network, southern California: implications for shear wave splitting and earthquake prediction, J. Geophys. Res. 95, 12449-12473.

Bai, C. Y., and B. L. N. Kennett (1999). Phase identification and attribute analysis of broadband seismograms at far-regional distances (submitted for publication).

Douglas, A. (1997). Bandpass filtering to reduce noise on Seismograms: is there a better way? Bull. Seism. Soc. Am. 87, 770-777. 


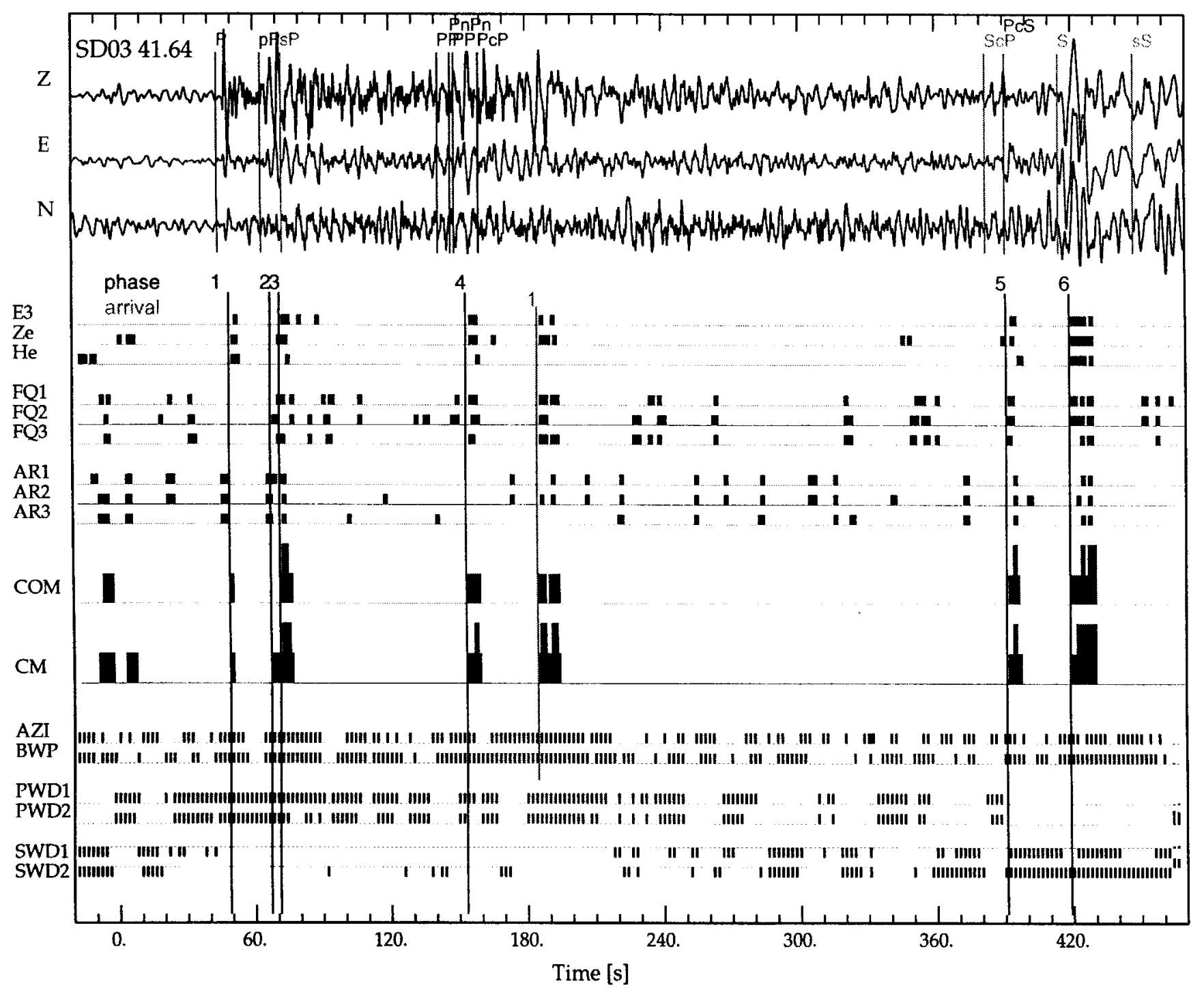

Figure 7. A further example of the application of the combination algorithms to event 4 for station SD03 at an epicentral distance of $41.64^{\circ}$. The traces follow Figure 1, with indications of clear phase detections (phases) and unassociated detections (arrivals).

Earle, P., and P. M. Shearer (1994). Characterization of global seismograms using an automatic-picking algorithm, Bull. Seism. Soc. Am. 84, 366376.

Kennett, B. L. N., and E. R. Engdahl (1991). Travel times for global earthquake location and phase association, Geophys. J. Int., 105, 429-465.

Leonard, M., and B. L. N. Kennett (1999). Multi-component auto-regressive techniques for the analysis of seismograms, Phys. Earth Planet. Inter. 113, 247-263.

Takanami, T., and G. Kitagawa (1988). A new efficient procedure for the estimation of onset times of seismic waves, J. Phys. Earth. 36, 267290.

Takanami, T., and G. Kitagawa (1993). Multivariate time-series models to estimate the arrival times of $S$ waves, Computers and Geosciences 19, 295-301.

Tong, C., and B. L. N. Kennett (1996). Automatic seismic event recognition and later phase identification for broadband seismograms, Bull. Seism. Soc. Am. 86, 1896-1909.
Vidale, J. E. (1986). Complex polarization analysis of particle motion, Bull. Seism. Soc. Am. 76, 1393-1405.

Wagner, G., and T. Owens (1996). Signal detection using multi-channel seismic data, Bull. Seism. Soc. Am. 86, 221-231.

Withers, M., R. Aster, C. Young, J. Beiriger, M. Harris, S. Moore, and J. Trujillo (1998). A comparison of select trigger algorithms for automated global seismic phase and event location. Bull. Seism. Soc. Am. 88, 95-106.

Research School of Earth Sciences

Australian National University

Canberra ACT 0200, Australia

(C.B., B.L.N.K.)

Manuscript received 22 September 1999. 